\title{
Technical Feasibility of Robotic Single Site Central Pancreatectomy with Duct-to-Mucosa Pancreaticojejunostomy in Cadaveric Experiment
}

\author{
Jin Ho Lee, M.D. ${ }^{2}$, Chang Moo Kang, M.D., Ph.D. ${ }^{1}$, Woo Jung Lee, M.D., Ph.D. ${ }^{1}$ \\ ${ }^{1}$ Department of Surgery, Yonsei University College of Medicine, Pancreaticobiliary Cancer Clinic, Yonsei Cancer Center, Severance Hospital, Seoul, \\ ${ }^{2}$ Department of Surgery, National Health Insurance Service Ilsan Hospital, Goyang, Korea
}

\begin{abstract}
Purpose: Laparoscopic single site surgery is currently available, but may not be feasible for delicate and complex surgical procedures. However, computer technology embedded into robotic surgical system could provide the way to advanced laparoscopic single site surgery.

Methods: 86-year-old, female cadaver who died from sepsis was used for testing technical feasibility of robotic single site surgical system (the da Vinci Surgical System_(Intuitive Surgical, Sunnyvale, CA)) in performing central pancreatectomy.
\end{abstract}

Results: About $4 \mathrm{~cm} \times 3 \mathrm{~cm} \times 1.5 \mathrm{~cm}$ sized segment of pancreatic neck portion was resected. Distal remnant pancreas was managed by two-layered, duct-to-mucosa pancreaticojejunostomy by intracorporeal suture technique. Operative procedure was completed in $150 \mathrm{~min}$.

Conclusion: Robotic single site central pancreatectomy with pancreaticojejunostomy was technically feasible in the present cadaveric experiment.

Keywords: Robotic single site operation, Central pancreatectomy, Duct to mucosa

Supplementary video file: This article contains supplementary material (https://doi.org/10.7602/ jmis.2016.19.4.162).

This is an Open Access article distributed under the terms of the Creative Commons Attribution Non-Commercial License (http:// creativecommons.org/licenses/by-nc/4.0/) which permits unrestricted non-commercial use, distribution, and reproduction in any medium, provided the original work is properly cited.
Received October 26, 2016

Revised November 21, 2016

Accepted November 21, 2016

\author{
Corresponding author \\ Chang Moo Kang \\ Department of Surgery, Yonsei \\ University College of Medicine, \\ Pancreaticobiliary Cancer Clinic, \\ Yonsei Cancer Center, Severance \\ Hospital, 50-1 Yonsei-ro, \\ Seodaemun-gu, Seoul 03722, Korea \\ Tel: +82-2-2228-2135 \\ Fax: +82-2-313-8289 \\ E-mail: cmkang@yuhs.ac
}

\section{INTRODUCTION}

In benign and borderline malignant tumor of the pancreas, function-preserving minimally invasive pancreatectomy is ideal approach. ${ }^{1,2}$ Laparoscopic single site surgery is currently available, but may not be feasible for delicate and complex surgical procedures. ${ }^{3,4}$ However, computer technology embedded into robotic surgical system could provide the way to advanced laparoscopic single site surgery. ${ }^{5}$

\section{MATERIALS AND METHODS}

86-year-old, female cadaver who died from sepsis was used for testing technical feasibility of robotic single site surgical system (the da Vinci Surgical System_(Intuitive Surgical, Sunnyvale, CA)) in performing central pancreatectomy. $\mathrm{Pa}-$ tient was supine position on the surgical table with $15^{\circ}$ re- $^{-}$ verse Trendelenburg position (Fig. 1A). Trans-umbilical skin incision for trocar was made by the approximately $3 \mathrm{~cm}$ skin incision in the umbilicus area (Fig. 1B) and specially designed 


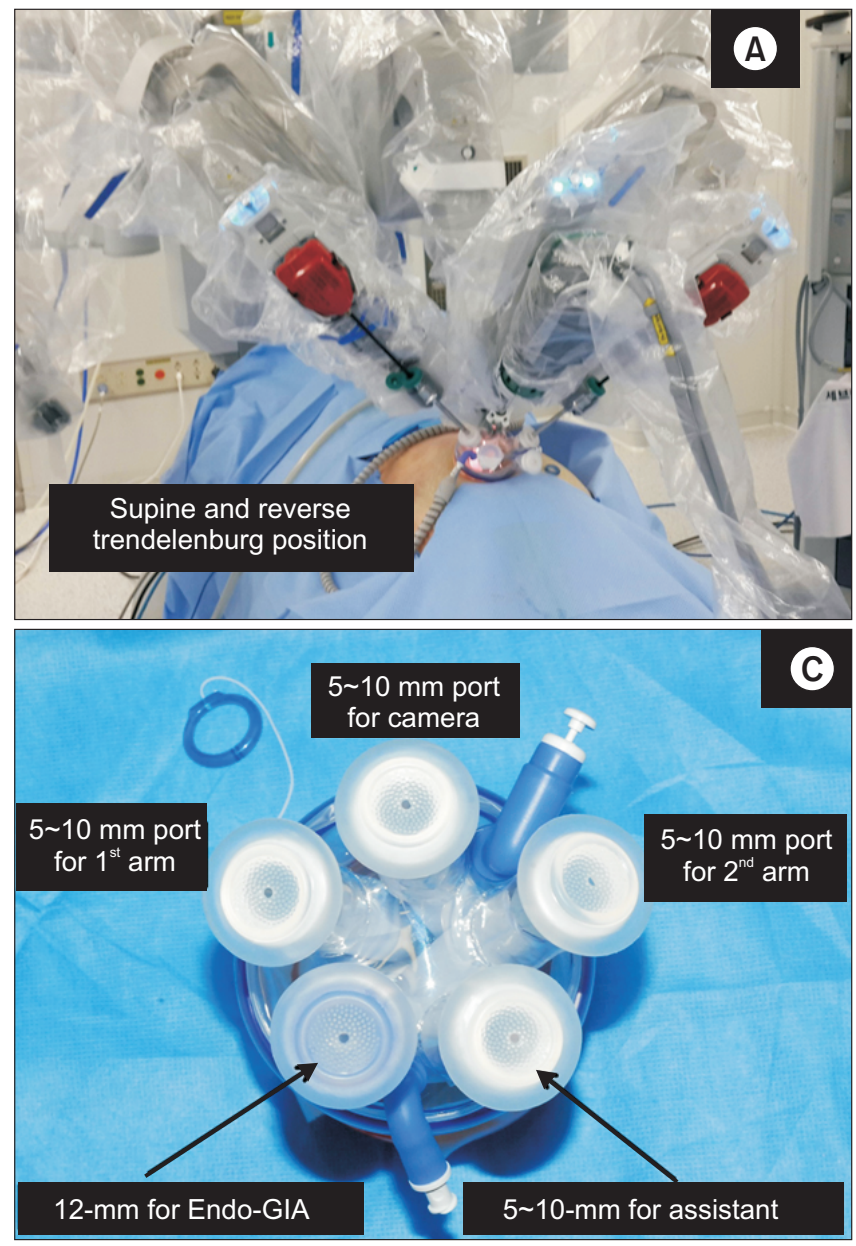

and modified glove port ${ }^{6}$ (NELIS, Bucheon, Gyeonggi-do) [not commercialized] with 5 fingers was inserted (Fig. 1C). The first arm of the robot was located on the right side of the patient and the second arm was positioned on the left side of the patient. And the camera was positioned in the middle of the two arms. Two $300 \mathrm{~mm}$-curved cannula was used, and specially designed port was applied for facilitating operative procedure, which makes it possible for assist surgeon to freely adjust length of robotic arm, introduce Tri-staple technology $600 \mathrm{~mm}$ medium/thick Endo-GIA Universal stapler, suture materials, and gauze without additional accessory port. Tributary vessels around pancreas were dissected and controlled by using crocodile grasper, mono-polar hook, dissector, and hem-o-lock clips.

\section{RESULTS}

About $4 \mathrm{~cm} \times 3 \mathrm{~cm} \times 1.5 \mathrm{~cm}$ sized segment of pancreatic neck portion was resected. Distal remnant pancreas was managed by two-layered, duct-to-mucosa pancreaticojejunostomy by intracoporeal suture technique. Operative procedure was com-

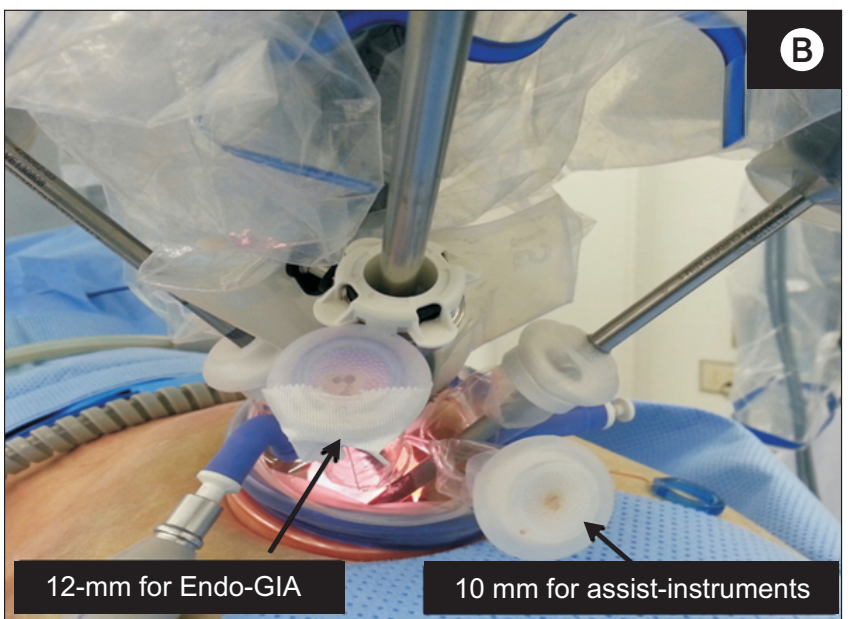

Fig. 1. Patient position and port placement. (A) Patient position: supine and reverse Trendelenburg position, (B) Port placement: Trans-umbilical area, (C) Specially designed modified glove port.

pleted in $150 \mathrm{~min}$.

\section{DISCUSSION}

The first central pancreatectomy (CP) performed in 1982 by Dagradi and Serio in patients with insulinoma of the pancreatic neck. After then, in 2004, Giluianotti et al. ${ }^{7}$ performed a robotic assisted $\mathrm{CP}$. According to pervious article we have published, ${ }^{1}$ more than 10 studies have been published on minimally invasive $\mathrm{CP}$, and about 60 patients have undergone minimally invasive $\mathrm{CP}$, suggesting the technical feasibility and safety. ${ }^{1,7}$ According to robotic technology and instruments have continuously developed, recently, there have been attempted single site robotic surgery. ${ }^{4,5}$

Nowadays, there has been single site laparoscopic surgery is performed in various abdominal surgery.9 But, especially in pancreatic surgery there is no single site laparoscopic surgery. Because the minimally invasive pancreatic surgery is needed more technically demanding procedure. In this aspect, robotic technology and instruments is useful for minimally invasive pancreatic surgery. But, some expert laparoscopic 
pancreatic surgeons think that laparoscopic approach for pancreatic disease is enough but robotic surgery is helpless for pancreatectomy. However, for some pancreatic surgeons who want to perform laparoscopic pancreatectomy without enough laparoscopic skills, robotic surgery can enable them to access pancreatic operation more easily. And in spite of lacks the endo-wrist technology current DaVinci robotic single site system is acceptable for the CP. In this regard, robotic surgery can enable them to access single site laparoscopic CP easily.

In conclusion, robotic single site central pancreatectomy with pancreaticojejunostomy was technically feasible in the present cadaveric experiment. Instrumental development, such as multifunctional single port and articulating robotic instruments will make this surgical technique much easier and feasible even in real human patients with benign and borderline malignant tumor near the neck of the pancreas. ${ }^{1,10}$

\section{ACKNOWLEDGMENTS}

We are thanks for NELIS ${ }^{\circledR}$ who produce a specially designed and modified glove port we demanded.

\section{REFERENCES}

1) Kang CM, Lee JH, Lee WJ. Minimally invasive central pancreatectomy: current status and future directions. J Hepatobiliary Pancreat Sci 2014;21:831-840.

2) Choi SH, Hwang HK, Kang CM, Yoon CI, Lee WJ. Pylorus- and spleen-preserving total pancreatoduodenectomy with resection of both whole splenic vessels: feasibility and laparoscopic application to intraductal papillary mucin-producing tumors of the pancreas. Surg Endosc 2012;26:2072-2077.

3) Spinoglio G, Lenti LM, Maglione V, et al. Single-site robotic cholecystectomy (SSRC) versus single-incision laparoscopic cholecystectomy (SILC): comparison of learning curves. First European experience. Surg Endosc 2012;26:1648-1655.

4) Levic K, Donatsky AM, Bulut O, Rosenberg J. A Comparative Study of Single-Port Laparoscopic Surgery Versus RoboticAssisted Laparoscopic Surgery for Rectal Cancer. Surg Innov 2015;22:368-375.

5) Scheib SA, Fader AN. Gynecologic robotic laparoendoscopic single-site surgery: prospective analysis of feasibility, safety, and technique. Am J Obstet Gynecol 2015;212:179 e171-178.

6) Choi SH, Hwang HK, Kang CM, Lee WJ. Transumbilical single port laparoscopic adrenalectomy: a technical report on right and left adrenalectomy using the glove port. Yonsei Med J 2012;53:442-445.

7) Giulianotti PC, Sbrana F, Bianco FM, Addeo P, Caravaglios G. Robot-assisted laparoscopic middle pancreatectomy. J Laparoendosc Adv Surg Tech A 2010;20:135-139.

8) Fung AK, Aly EH. Systematic review of single-incision laparoscopic colonic surgery. Br J Surg 2012;99:1353-1364.

9) Rabischong B, Compan C, Savary D, et al. [Laparoendoscopic single-site surgery in gynecology: the situation in 2013]. J Gynecol Obstet Biol Reprod (Paris) 2013;42:445-457.

10) Kang CM, Kim DH, Lee WJ, Chi HS. Initial experiences using robot-assisted central pancreatectomy with pancreaticogastrostomy: a potential way to advanced laparoscopic pancreatectomy. Surg Endosc 2011;25:1101-1106. 